\title{
Progress and Challenges in Clinical AAV Gene Therapy for Neurological and Neuromuscular Disorders
}

\author{
Megan Baird ${ }^{1,2 *}$, Maura Schwartz ${ }^{1,2 *}$, Kathrin Meyer ${ }^{1,2,3 * *}$ and Nicolas Wein ${ }^{1,2,3 * *}$ \\ ${ }^{1}$ Center for Gene Therapy, Abigail Wexner Research Institute at Nationwide Children's Hospital, USA \\ ${ }^{2}$ Biomedical Sciences Graduate Program, The Ohio State University College of Medicine, USA \\ ${ }^{3}$ Department of Pediatrics, The Ohio State University, USA \\ *Megan Baird and Maura Schwartz contributed equally to this work.
}

*Corresponding author: Kathrin Meyer and Nicolas Wein, The Center for Gene Therapy, The Research Institute, Nationwide Children's Hospital 700 Children's Drive, Columbus, Ohio

\begin{abstract}
The field of gene therapy is growing at a rapid pace. The discovery and modification of adeno-associated viruses (AAV) as gene therapy vectors has allowed for the development of novel treatments for neurological and neuromuscular disorders. AAV based gene therapy techniques can be utilized to add, replace, or modify genes and their expression in patients, thus providing a potential beneficial therapeutic effect. In many cases, gene therapy has the promising power to change the natural course of debilitating and fatal diseases and give patients a new outcome on life. This mini review will give a brief overview of the AAV gene therapy field, highlighting FDA approved gene therapies and selected current in-clinic AAV-mediated therapeutics for neurological and neuromuscular diseases for which interim data are available. These therapies and the ongoing clinical trials are currently laying the groundwork for future gene therapy strategies and clinical trial design. Ultimately, they will inform the field on promises and limitations that still need to be overcome.
\end{abstract}

Keywords: Gene Therapy; AAV; Neuromuscular Disorders

Abbreviations: AAV- Adeno-associated virus; RAAV- Recombinant adeno-associated virus; ITRs- Inverted terminal repeats. FDA-Food and Drug Administration; CNS-Central nervous system; IV- Intravenous; NCL-Neuronal ceroid lipofuscinosis; CSF- Cerebrospinal fluid; IT- Intrathecal; PDParkinson's Disease; ALS- Amyotrophic Lateral Sclerosis; DMD- Duchenne Muscular Dystrophy

\section{Introduction}

Adeno-associated viruses (AAV) are small, replicationdefective, nonenveloped viruses from the family Parvoviridae. First discovered in the mid-1960s, AAV was thought to be a contaminant in adenovirus cultures [1,2]. Over the next 15-20 years, studies to understand basic AAV biology began, characterizing AAV elements such as genome composition and configuration [1,2]. AAV contains a linear single-stranded DNA genome containing approximately 4.7 kilobases [2,3]. As AAV is non-integrating and has a low immunogenic profile, it has become an attractive candidate for viral mediated gene therapies following the unfortunate death of Jesse Gelsinger in 1999. Jesse's death was caused by immune complications triggered by an adenoviral based gene therapy. Despite the hesitation and fear that resulted from this event, the advent of recombinant AAV (rAAV), showing a lower immunogenic profile compared to adenoviruses, was able to propel the field forward. The first rAAV clinical trial was initiated in 1995 as a treatment for cystic fibrosis [4]. In the early 2000s, new AAV subtypes (serotypes) with the capability to infect various tissues and cell types were discovered in primates, leading to an expansion of the AAV in vivo gene delivery toolbox [2]. The intent of this mini review is to give a brief overview of current Food and Drug Administration (FDA) approved AAV gene therapies as well as to highlight additional AAV vectors currently in clinical trials for neurological and neuromuscular disorders. Due to large number of clinical trials, the mini review will only focus on 
selected programs for which interim data has been made public, as well as on showcasing different gene therapy methods including not only classic gene replacement strategies but also gene expression (mRNA) modulation [5,6]. Moreover, we will shortly discuss the limitations and current hurdles of AAV based therapeutics.

\section{FDA approved AAV Gene Therapies}

RPE65 mutation-associated retinal dystrophy: Fifty-two years after the initial discovery of AAV, the FDA approved the first gene replacement strategy for treatment of a monogenic disorder $[7,8]$. Voretigene neparvovec-rzyl, or Luxturna, was approved in December 2017 to treat patients with confirmed biallelic RPE65 mutation-associated retinal dystrophy [9]. RPE65 is essential for regeneration of the visual pigment necessary for photoreceptor mediated vision [10]. Without regeneration, the unconverted pigment builds up within the retinal pigment epithelium, blocks the visual cycle, and leads to visual impairment especially in lowlight conditions [10]. Luxturna prevents and partially reverses vision loss by restoring expressing of the RPE65 gene delivered to the retinal pigment epithelium via subretinal injection of AAV2 $[8,10] .55 \%$ of patients treated in the initial clinical trial were able to navigate an obstacle course at 2 light levels darker compared to testing prior to treatment [8]. The approval of Luxturna demonstrated safety, utility, and effectiveness of AAV mediated gene replacement strategies. However, the site of administration served as an advantage in this case since the eye is an immune-privileged site [11]. For gene therapies targeted to organs outside the eye, the innate immune response can become a greater obstacle.

Spinal Muscular Atrophy: The first intravenous (IV) delivered AAV9 gene therapy was approved by the FDA in 2019 for treatment of infants below 2 years of age suffering from Spinal Muscular Atrophy [9]. Loss of the SMN1 gene is detrimental to motor neuron survival and leads to whole body muscle weakness [12]. Death typically occurs by two years of age due to respiratory failure [13]. Onasemnogene abeparvovec-xioi (Zolgensma) is an AAV9 based gene therapy that contains the coding sequence of the SMN protein [14]. Treatment allowed SMA patients to achieve new motor milestones, like sitting without assistance, and successfully prevents early death [15]. The first treated patients are now five years of age [15]. Zolgensma's market approval was a landmark success for the SMA field and solidified the idea for translation of AAV based gene replacement strategies as an effective option for other neuromuscular disorders. Additional clinical trials are currently testing Onasemnogene abeparvovec-xioi in older patients using intrathecal delivery of the vector into the cerebrospinal fluid (CSF). To date, Voretigene neparvovec-rzyl and Onasemnogene abeparvovec-xioi are the only FDA approved AAV gene therapies on the market, but over 100 clinical trials are currently ongoing using different AAVs and delivery routes for a wide range of diseases.
Selected ongoing clinical trials for neurological and neuromuscular disorders with published interim data

Batten disease: Batten Disease encompasses a number of neuronal ceroid lipofuscinoses (NCL) disorders which mostly affect children [16]. Mutations in CLN genes generally result in accumulation of lipofuscin granules in neuronal cells and lead to blindness, regression in cognitive and intellectual ability, seizures, loss of motor function, and early death [16]. Nationwide Children's Hospital (Columbus, OH, USA) developed gene therapy approaches for Batten Disease CLN6 and CLN3 which were later licensed by Amicus Therapeutics. Both Phase I/II gene replacement clinical trials are currently ongoing [17]. For CLN6 Batten Disease, AAV9. CLN6 (AT-GTX-501) is injected directly at a dose of $1.5 \mathrm{e} 13 \mathrm{vg} / \mathrm{kg}$ into the cerebrospinal fluid (CSF) via lumbar intrathecal delivery [17]. As of November 2020, efficacy data from the 24-month follow-up visit has been reported for eight children [18]. Compared to subjects from the natural history study, who had a mean rate of decline of 2.4 points over 24 months, treated patients had a mean rate of decline of only 0.6 points on the Hamburg motor and language scale [18]. Amicus also reported safety data that showed treatment was well-tolerated [18]. The treatment for CLN3 Batten Disease with AAV9.CLN3 (AT-GTX-502) began in late 2018, but sufficient interim data has yet to be reported [19]. In addition to these therapies, several ongoing or upcoming clinical studies are being dedicated to treatment of CLN2 Batten Disease, including those from Weill Cornell Medical School (AAVrh10.CLN2), Spark Therapeutics (SPK-1001), and REGENXBIO (RGX-181) [20-22].

Duchenne muscular dystrophy (DMD): DMD is an X-linked recessive disorder characterized by severe muscle weakness and loss of ambulation affecting children around the ages of 8-13 [23]. The disease later progresses to respiratory complications, cardiac abnormalities, and eventual death [23]. The disorder is caused by mutations in the DMD gene, which encodes the dystrophin protein [23]. Without dystrophin, the plasma membrane (sarcolemma) of muscle cells becomes unstable which leads to muscle degeneration and myofiber loss [24]. Unfortunately, the DMD mRNA is too big and therefore cannot be packaged into AAV vectors [25]. However, in 1990, a researchers described a milder form of muscular dystrophy in patients expressing a truncated form of the protein that still had functional properties [25]. As a result, researchers began developing truncated dystrophin proteins, termed micro and mini dystrophin, that could be packaged into AAV and still have therapeutic effects [25]. Three micro-dystrophin constructs are currently being tested in clinical trials: Sarepta Therapeutics (rAAVrh74; SRP-9001), Pfizer (AAV9), and Biosciences (AAV9) [26-28]. All constructs were delivered intravenously and in varying doses ranging from $5 \mathrm{e} 13$ $\mathrm{vg} / \mathrm{kg}$ to $2 \mathrm{e} 14 \mathrm{vg} / \mathrm{kg}[23,29,30]$. Overall, the treatments were well tolerated with the exception of the Biosciences trial, which was 
placed on hold several times due to concerns of severe complement activation [23,29-31]. However, all treatments were shown to be efficacious with reports of decreased creatine kinase (CK) levels and improvements in North Star Ambulatory Assessment scores [23,29-31]. Notably, the efficacy seems to be dose-dependent with $74.3 \%-95.8 \%$ of muscle fibers expressing the new dystrophin protein after delivery of 2e14vg/kg micro-dystrophin in Sarepta's trial $[9,23]$.

In addition to the micro- and mini-dystrophin trials, another AAV clinical trial for DMD was initiated this year, taking advantage of the modularity of this giant protein using mRNA exon skipping technology. This approach can be used to treat a subpopulation of DMD patients in which exon 2 of the gene is duplicated. The AAV9 therapeutic vector contains a small RNA that binds to the dystrophin messenger RNA (mRNA) and alters mRNA splicing to promote exon exclusion. Exon duplications encompass approximately $10-15 \%$ of disease-causing mutations in DMD, with exon 2 being the most commonly duplicated exon ( 1-2\% of DMD patients) $[32,33]$. Exclusion of exon 2 results in production of either mRNA containing a single copy of exon 2 and production of normal dystrophin protein; or mRNA with no copies of exon 2, resulting in a highly functional shorter isoform of the dystrophin protein [34]. The phase I/II clinical trial is currently ongoing using IV injection of $3 \mathrm{e} 13 \mathrm{vg} / \mathrm{kg}$ with this vector for treatment of DMD patients carrying exon 2 duplications. The primary outcome measure is safety and secondary outcomes include monitoring change in dystrophin expression and changes in exon 2 inclusion in the mRNA transcript of dystrophin [35]. Interim data from 3 months post injection was recently presented at the World Muscle Society meeting showing reduction of creatine kinase levels as well as increased dystrophin protein expression in treated patients.

X-linked myotubular myopathy (XLMTM): Mutations in the MTM1 gene, encoding myotubularin, causes XLMTM which results in extreme muscle weakness, respiratory failure, and death [36]. Audentes Therapeutics is conducting a Phase I/II clinical trial investigating the treatment of patients less than five years of age with an AAV8.hMTM1 (AT132) construct delivered intravenously [37]. A low dose $(1 \mathrm{e} 14 \mathrm{vg} / \mathrm{kg})$ and a high dose $(3 \mathrm{e} 14 \mathrm{vg} / \mathrm{kg})$ were delivered [37]. As of May 2019, safety and efficacy data was available for six patients given the low dose and four patients given the high dose [38]. All patients showed improved motor function including the ability to sit unassisted, raise self to stand, and walk with or without support [38]. In addition, time of ventilator assistance was decreased with most patients becoming fully independent [38]. These milestones persisted to over 1-year post treatment and were consistent with increased expression of myotubularin in muscle biopsies [38]. Based on these initial highly promising results, the $3 \mathrm{e} 14 \mathrm{vg} / \mathrm{kg}$ dose was chosen for the confirmatory phase [38]. To date, a total of 17 patients received the high dose, which unfortunately led to severe side effects and death in three patients. Preliminary findings indicate that the immediate cause of death was sepsis. Although the exact mechanisms that led to these deaths remain unknown, the patients who suffered severe side effects were amongst the older and heavier patients, therefore receiving an overall larger dose of viral vector since dosing is calculated by kilogram body weight. Moreover, the patients had pre-existing hepatobiliary disease, which could have increased their sensitivity to the treatment since the liver is highly targeted by AAVs $[39,40]$.

Limb girdle muscular dystrophy (LGMD): Limb Girdle Muscular Dystrophies (LGMD) are a distinct class of muscular dystrophies with 34 identified variants, each showing significant phenotypic heterogeneity [41]. LGMD Type 2E (LGMD2E) is a childhood to adolescent onset LGMD characterized by progressive weakness in the pelvic-girdle and shoulder-girdle muscles, typically resulting from mutations in the beta-sarcoglycan gene (SGCB) [42]. Sarepta Therapeutics has an ongoing AAV-mediated gene replacement clinical trial for the treatment of patients with LGMD2E [43]. In this trial, cohort 1 patients, 4-15 years of age with a confirmed beta-sarcoglycan gene mutation in both alleles, received a single IV injection of AAVrh74 containing human SGCB cDNA under the control of the muscle specific MHCK7 promoter (AAVrh74.MHCK7. SGCB) at the dose of 5e13vg/kg [43,44]. Muscle biopsies taken 3 months post-treatment from 3 patients show an average of 51\% SGCB positive muscle fibers, a $90 \%$ reduction in CK levels, and restoration of the sarcoglycan complex to the membrane $[25,44]$. At the 90 -day patient follow-up, 2 patients had elevated liver enzymes as a side effect of the AAV treatment, which resolved with supplemental steroid treatment [44]. Cohort 2 patient dosing will be based on results from cohort 1 patients and may be escalated [43].

Parkinson's disease (PD): Parkinson's Disease (PD) is an adult-onset neurodegenerative disease with motor and cognitive impairment resulting from the loss of striatal dopaminergic neurons in the substantia nigra of the brain. In PD, motor impairment manifests as bradykinesia, tremor, and rigidity. Non-motor impairments include cognitive, mood, and behavioral dysfunction, sleep disturbance, pain, and autonomic disturbances [45]. The most effective pharmacological treatment for PD is levodopa, a dopamine precursor amino acid, but as the disease progresses, the benefits of levodopa treatment decline due to the loss of Aromatic L-Amino Acid Decarboxylase (AADC), an enzyme that converts levodopa to dopamine [46]. Neurocrine Biosciences, in collaboration with University of California San Francisco, Feinstein Institute for Medical Research, Oregon Health and Science University, and Voyager Therapeutics, recently completed a clinical trial safety study of AADC gene therapy as a treatment for PD [47]. In this study, fifteen patients received bilateral putamen injections of AAV2 containing AADC cDNA (VY-AADC01) at the dose of 7.5e11vg ( $\mathrm{n}=5), 1.5 \mathrm{e} 12 \mathrm{vg}$ $(n=5)$, or 4.7e12vg $(n=5)$ [47]. Results from this study show a dose dependent putaminal coverage of VY-AADC01 expression and dose dependent increases in AADC activity, as well as a reduction of ant parkinsonism medications at 6 months [46]. The Unified Parkinson's Disease Rating Scale Part III (UPDRS-III) was used to evaluate changes in motor and non-motor complications and showed clinically meaningful dose-dependent improvements at 12 months and increased Quality of Life measurements even with 
reductions in dopaminergic medications [46,47]. A Phase II clinical study (NCT03562494) has been recently initiated, sponsored by Neurocrine Biosciences in collaboration with Voyager Therapeutics and is currently recruiting [48].

Amyotrophic lateral sclerosis (ALS): ALS is an adult-onset neurodegenerative disease characterized by progressive loss of motor neurons in the brain and spinal cord. As there is currently no cure, the disease is always fatal with patients succumbing to death within 2-5 years of symptom onset. In July 2020, The New England Journal of Medicine published results from a compassionate-use patient study for the treatment of familial ALS. The study was conducted at University of Massachusetts and Massachusetts General Hospital and included treatment of two patients with SOD1mediated ALS [49]. The gene therapy construct contains a microRNA sequence that reduces expression of the superoxide dismutase 1 (SOD1) protein. Mutated forms of this protein cause approximately $2 \%$ of all ALS cases. Two patients were intrathecally injected with AAVrh10-miR-SOD1 at the dose of 4.2e14 vector genomes [49]. Patient 1 experienced an adverse inflammatory response to the viral vector causing severe pain which decreased over time but was not resolved [49]. Patient 1 had transient improvements in the strength of his right leg after treatment, but no change in vital capacity, and succumbed to the disease 15.6 months post treatment [49]. The gene therapy treatment led to a $90 \%$ reduction of SOD1 protein in the post-mortem lumbosacral spinal cord of Patient 1 but there was no change in SOD1 levels in the cerebrospinal fluid with treatment [49]. Western Immunoblot analysis of SOD1 protein in the spinal cord showed no differences in the cervical spinal cord, though there was a reduction in SOD1 enzyme activity [49]. For Patient 2, a more rigorous immunosuppressive regimen was used to reduce chances of a strong inflammatory response and the patient did not have increased hepatic aminotransferase levels or sensory dysfunction following treatment [49]. As patient 2 has a milder form of the disease and a slower disease progression, additional data on the disease course is expected to be collected over time.

\section{Conclusion}

The field of AAV mediated gene therapy has grown exponentially over the last decade, with a massive increase in publications, preclinical studies, and ongoing or completed clinical trials [2]. Though many results so far are exciting, these clinical studies also underline challenges the field is still attempting to overcome. In all the aforementioned trials, pre-existing immunity, measured in form of antibodies, were designated as exclusion criteria. This is a significant barrier to treatment for patients in need, and much of the field is dedicated to finding strategies that can temporarily evade or dampen the immune response of patients that have been naturally exposed to AAV viruses [9,50]. These strategies, such as the use of immunomodulatory enzymes, plasmapheresis, or modified AAV capsids, may also be useful in the event patients need a second administration of vector to maintain expression of the transgene in cell types that continue to divide and therefore might lose the AAV construct over time [9]. For the treatment of neurological and neuromuscular diseases, choosing an appropriate route of administration is vital to the success of a therapy. Local routes of delivery, such as direct brain injections, allow for sufficient local targeting but increase the risk of injury at the site of injection [51]. Several of the described studies are currently utilizing IV delivery of AAV constructs capable of targeting muscles but also brain and spinal cord. The caveat of IV delivery is that high doses are required for sufficient transduction of target cell types, especially in the central nervous system (CNS). As a result, several studies have reported serious adverse events related to liver toxicity, an organ that is highly targeted with IV delivery [39,52,53]. Alternatively, CSF delivery via intrathecal (IT) route offers several advantages over IV delivery for neurological disorders because it allows for sufficient transduction of neuronal cell types with a lower required dose, thereby limiting immunogenic response and liver targeting [54]. The CSF delivery route could potentially lead to toxicity in other organs, as a recent study performed in non-human primates suggests this route may induce dorsal root ganglion (DRG) pathology [55]. To date, DRG pathology or clinical signs thereof have not been reported in patients dosed intrathecally in the ongoing Batten disease trials or the clinical trials currently conducted with Zolgensma for older patients (NCT03381729). Another consideration is when to dose patients for maximal therapeutic effect. In general, it seems the highest benefit is achieved in patients that are dosed early in disease progression with as few symptoms as possible, as symptom reversion seems to be more challenging. The currently ongoing clinical trials and newly initiated studies will be highly informative and will help advance the exciting field of AAV gene therapy further.

\section{Acknowledgements}

This review was written by MB, MS, KM and NW Research in the Meyer and Wein lab on projects related to this topic NIH 5R01AR073908-02 and industry partners, M.B. is also supported by the OTA graduate student award.

\section{Conflict of Interest}

KM and NW Royalties from intellectual property licensed by Amicus Therapeutics and Audentes Therapeutics.

\section{References}

1. Hastie E, Samulski RJ (2015) Adeno Associated Virus at 50: A Golden Anniversary of Discovery, Research, and Gene Therapy Success-A Personal Perspective. Hum. Gene Ther 26(5): 257-265.

2. Wang D, Tai PWL, Gao G (2019) Adeno-associated virus vector as a platform for gene therapy delivery. Nat Rev Drug Discov 18(5): 358-378.

3. Naso MF, Tomkowicz B, Perry WL, Strohl WR (2017) Adeno-Associated Virus (AAV) as a Vector for Gene Therapy. Bio Drugs 31(4): 317-334.

4. Flotte T, Carter B, Conrad C, Guggino W, Reynolds T, et al. (1996) A phase I study of an adeno-associated Virus-CFTR gene vector in adult CF patients with mild lung disease. Hum. Gene Ther 7(9): 1145-1159.

5. Domenger C, Grimm D (2019) Next-generation AAV vectors-do not judge a virus (only) by its cover. Hum Mol Genet 28: R3-R14.

6. He X, Xie H, Liu X, Gu F (2019) Basic and Clinical Application of AdenoAssociated Virus-Mediated Genome Editing. Hum Gene Ther 30(6): 673681. 
7. Luxturna|Fda.

8. Fda Highlights of Prescribing Information: Luxturna.

9. Prasad R, Minter M, Lugo T, Corwin S, Hanbury Brown L, et al. (2020) It's All in the Delivery: Understanding Viral and Nonviral Approaches to In Vivo Gene Therapy and Gene Editing. William Blair Ind Rep.

10. Cai X, Conley SM, Naash MI (2009) RPE65: Role in the visual cycle, human retinal disease, and gene therapy. Ophthalmic Genet 30(2): 57-62.

11. Niederkorn JY (2019) The eye sees eye to eye with the immune system: The 2019 Proctor lecture. Investig Ophthalmol Vis Sci 60(13): 44894495.

12. Farrar MA, Kiernan MC (2015) The Genetics of Spinal Muscular Atrophy: Progress and Challenges 12(2): 290-302.

13. National Institute of Neurological Disorders and Stroke Spinal Muscular Atrophy Fact Sheet.

14. Fda Cber Package Insert - ZOLGENSMA.

15. Novartis Zolgensma ${ }^{\circledR}$ data shows rapid, significant, clinically meaningful benefit in SMA including prolonged eventfree survival, motor milestone achievement and durability now up to 5 years post-dosing.

16. Johnson TB, Cain JT, White KA, Ramirez Montealegre D, Pearce DA, et al. (2019) Therapeutic landscape for Batten disease: current treatments and future prospects. Nat Rev Neurol 15: 161-178.

17. Clinicaltrials.gov Batten CLN6 Gene Therapy (2016).

18. De Los Reyes E, Meyer K, Lehwald L, Albright C, Rogers DL, et al. (2016) Single-dose AAV9-CLN6 gene transfer stabilizes motor and language function in variant late infantile neuronal ceroid lipofuscinosis 6 (vLINCL6) disease: interim results from the first clinical gene therapy trial Background and Objectives.

19. (2020) Clinicaltrials.gov Gene Therapy for Children with CLN3 Batten Disease.

20. Safety Study of a Gene Transfer Vector (Rh.10) for Children with Late Infantile Neuronal Ceroid Lipofuscinosis (LINCL).

21. (2020) Spark Therapeutics Announces Presentation of Preclinical Data in Pompe Disease and CLN2 Disease at $15^{\text {th }}$ Annual WORLDSymposiumTM - Spark Therapeutics.

22. (2020) FDA Grants Orphan Drug Designation To RGX-181 Gene Therapy for The Treatment of CLN2 Form of Batten Disease | REGENXBIO Inc.

23. Mendell JR, Sahenk Z, Lehman K, Nease C, Lowes LP, et al. (2020) Assessment of Systemic Delivery of rAAVrh74.MHCK7. micro-dystrophin in Children with Duchenne Muscular Dystrophy: A Nonrandomized Controlled Trial. JAMA Neurol 77(9): 1122-1131.

24. Gao QQ, McNally EM (2015) The dystrophin complex: Structure, function, and implications for therapy. Compr Physiol 5(3): 1223-1239.

25. Crudele JM, Chamberlain JS (2019) AAV-based gene therapies for the muscular dystrophies. Hum Mol Genet 28(R1): R102-R107.

26. (2020) Microdystrophin Gene Transfer Study in Adolescents and Children with DMD.

27. (2020) Systemic Gene Delivery Clinical Trial for Duchenne Muscular Dystrophy (DMD).

28. A Study to Evaluate the Safety and Tolerability of PF-06939926 Gene Therapy in Duchenne Muscular Dystrophy (2020)

29. (2020) Pfizer Inc (2020) Pfizer's New Phase 1b Results of Gene Therapy in Ambulatory Boys with Duchenne Muscular Dystrophy (DMD) Support Advancement into Pivotal Phase 3 Study.

30. (2020) Solid Biosciences Provides Data Update from SGT-001 Development Program | Solid Biosciences Inc. Available online.

31. (2020) Solid Biosciences Presents Data from Duchenne Muscular Dystrophy Gene Therapy Program at ASGCT 23rd Annual Meeting | Solid Biosciences Inc. Available online.

32. White SJ, Aartsma Rus A, Flanigan KM, Weiss RB, Kneppers ALJ, et al. (2006) Duplications in the DMD gene. Hum. Mutat 27(9): 938-945.
33. Greer KL, Lochmüller H, Flanigan K, Fletcher S, Wilton SD (2014) Targeted exon skipping to correct exon duplications in the dystrophin gene. Mol Ther Nucleic Acids 3(3): e155.

34. Wein N, Vulin A, Falzarano MS, Szigyarto CAK, Maiti B, et al. (2014) Translation from a DMD exon 5 IRES results in a functional dystrophin isoform that attenuates dystrophinopathy in humans and mice. Nat Med 20(9): 992-1000.

35. AAV9 U7snRNA Gene Therapy to Treat Boys with DMD Exon 2 Duplications (2020) Full Text View - ClinicalTrials.gov Available online.

36. Shieh PB, Kuntz N, Smith B, Bönnemann CG, Dowling JJ, et al. (2017) 652-ASPIRO Phase 1/2 Gene Therapy Trial In X-Linked Myotubular Myopathy (XLMTM): Update on Preliminary Safety and Efficacy Findings.

37. Gene Transfer Clinical Study in X-Linked Myotubular Myopathy (2020) Full Text View - ClinicalTrials.gov Available online.

38. (2020) ASPIRO Phase 1/2 Gene Therapy Trial In X-Linked Myotubular Myopathy (XLMTM): Update on Preliminary Safety And Efficacy Findings | MDA Clinical \& Scientific Conference 2021 Available online.

39. (2020) Audentes Therapeutics Provides Update on the ASPIRO Clinical Trial Evaluating AT132 in Patients with X-linked Myotubular Myopathy | Business Wire Available online.

40. Audentes Therapeutics Letter to XLMTM Patient Community.

41. Nallamilli BRR, Chakravorty S, Kesari A, Tanner A, Ankala A, et al. (2018) Genetic landscape and novel disease mechanisms from a large LGMD cohort of 4656 patients. Ann Clin Transl. Neurol 5(12): 1574-587.

42. Pozsgai ER, Griffin DA, Heller KN, Mendell JR, Rodino Klapac LR (2017) Systemic AAV-Mediated $\beta$-Sarcoglycan Delivery Targeting Cardiac and Skeletal Muscle Ameliorates Histological and Functional Deficits in LGMD2E Mice. Mol Ther 25(4): 855-869.

43. Gene Delivery Clinical Trial of SRP-9003 for Patients with LGMD2E (Beta-sarcoglycan Deficiency) (2020) Full Text View - ClinicalTrials.gov Available online.

44. (No Title) Available online.

45. Poewe W, Seppi K, Tanner CM, Halliday GM, Brundin P, et al. (2017) Parkinson disease. Nat Rev Dis Prim 3: 1-21.

46. Christine CW, Bankiewicz KS, Van Laar AD, Richardson RM, Ravina B, et al. (2019) Magnetic resonance imaging-guided phase 1 trial of putaminal AADC gene therapy for Parkinson's disease. Ann. Neurol 85(5): 704-714.

47. Safety Study of AADC Gene Therapy (VY-AADC01) for Parkinson's Disease (2020) Full Text View - ClinicalTrials.gov Available online.

48. VY-AADC02 for Parkinson's Disease with Motor Fluctuations (RESTORE-1) (2020) Full Text View -ClinicalTrials.gov Available online.

49. Mueller C, Berry JD, McKenna Yasek DM, Gernoux G, et al. (2020) SOD1 suppression with adeno-associated virus and MicroRNA in familial ALS. N Engl J Med 383: 151-158.

50. Colella P, Ronzitti G, Mingozzi F (2018) Emerging Issues in AAV-Mediated In Vivo Gene Therapy. Mol. Ther. Methods Clin Dev 8: 87-104.

51. Gessler DJ, Tai PWL, Li J, Gao G (2019) Intravenous infusion of AAV for widespread gene delivery to the nervous system. In Methods in Molecular Biology. Humana Press Inc 1950: 143-163.

52. Hinderer C, Katz N, Buza EL, Dyer C, Goode T, et al. (2018) Severe Toxicity in Nonhuman Primates and Piglets Following High-Dose Intravenous Administration of an Adeno-Associated Virus Vector Expressing Human SMN. Hum Gene Ther 29(3): 285-298.

53. (2020) High dose AAV gene therapy deaths. Nat Biotechnol 38: 910.

54. Meyer K, Ferraiuolo L, Schmelzer L, Braun L, McGovern V, et al. (2015) Improving single injection CSF delivery of AAV9-mediated gene therapy for SMA: A dose-response study in mice and nonhuman primates. Mol Ther 23(3): 477-487.

55. Hordeaux J, Buza EL, Dyer C, Goode T, Mitchell TW, et al. (2020) AdenoAssociated Virus-Induced Dorsal Root Ganglion Pathology. Hum Gene Ther 31(15-16): 808-818 